\title{
Curriculum vitae: Helen Bygrave
}

Helen Bygrave has worked with Médecins Sans Frontières (MSF) in Myanmar, Nigeria, and southern Africa. She lives in London and continues to keep her hand in general practice

\section{Jacqui Wise}

London, UK

\section{Variety and flexibility}

Doing one specialty seven days a week wasn't for me, so I decided to go into general practice. I worked in Kentish Town in London and loved being a GP. I enjoyed the variety of the diagnostic work, the continuity with patients, and the flexibility. Alongside my GP work I continued as a clinical assistant doing breast oncology at the Mount Vernon Cancer Centre, and also got involved in teaching medical students at the practice.

\section{HIV}

In 2001, after finishing my GP training, I went to Uganda for six months and did HIV work for the first time. There was no national antiretroviral programme so it was mainly palliative care-treating opportunistic infections and providing symptom control.

\section{Lazarus effect}

After Uganda I worked as a salaried GP in north London for five years but got itchy feet. I applied to MSF and was offered an HIV project in Nigeria. The contrast with my time in Uganda was enormous-five years down the line we now had access to triple therapy antiretrovirals (ARVs). It was fantastic to see the "Lazarus effect"- people transformed within three to four months of treatment. I sometimes wouldn't recognise people in the corridors of the clinic; they had changed so much and they were able to start to rebuild their lives.

\section{Political restrictions}

I had originally applied to do just one year with MSF but I enjoyed it so much and learnt such a lot that I applied for another post-this time in Myanmar. There was a low prevalence of HIV in the country so we were mainly working with key populations-drug users, men having sex with men, and sex workers. We also had a project in Rakhine state working with the Rohingya population. It was a very restrictive political environment and MSF was the only provider of HIV care in the country.

\section{Lesotho}

By this time, I was hooked on HIV and MSF, and I applied for a third post in Lesotho in 2008. By now, there were national programmes providing ARVs but care was still very centralised. Sometimes patients had to travel up to four hours to get their drugs. During my time in Lesotho I got involved in more operational research as well as training, advocacy, and mentorship.

\section{Advisory role}

An advisor job became available at MSF's Southern Africa Medical Unit which I was lucky enough to get and ended up supporting projects in Kenya, Lesotho, Malawi, and Zimbabwe. At first I was based in Cape Town but then came back to London and combined the role with GP work. Over the past few years I have also carried out consultancy work for organisations such as the International AIDS Society, Primary Care International, the World Health Organization, and the Zimbabwe Ministry of Health.

\section{Clinical luxuries}

In between every mission for MSF I worked as a GP locum, including some out-of-hours work, and it was great to have that clinical time. I still work regularly for an out-of-hours provider based at Homerton University Hospital. I really enjoy the clinical work with the luxury of having access to diagnostic tests and the ear of a specialist.

\section{Access to treatment}

Since January I have been working for the MSF Access Campaign on non-communicable diseases. There are a lot of potential lessons to be learnt from the scale up of HIV treatment. With HIV, for example, the game changer was putting three drugs into one pill. We are now having the same conversations about combination drugs to help simplify the management of cardiovascular disease in resource poor settings. 


\section{Career Timeline}

2018 to date Non-communicable diseases advisor, MSF Access Campaign

2010 to date HIV and tuberculosis advisor, MSF

2015 to date Consultant, International AIDS Society

2010 to date GP, out-of-hours provider, City and Hackney, London

2008 to 2010 Medical coordinator, MSF Lesotho

2007 to 2008 HIV advisor for MSF, Myanmar

2005 to 2006 Medical doctor, MSF HIV programme, Lagos, Nigeria

2001-2005 GP, Kentish Town, London

1988-1995 Cambridge University and University College London School of Medicine 